\title{
An Iterative Domain Decomposition Method for the Solution of a Class of Indefinite Problems in Computational Structural Dynamics
}

\author{
Charbel Farhat ${ }^{\mathrm{a}, *}$ and Jing $\mathrm{Li}^{\mathrm{a}}$ \\ ${ }^{a}$ Department of Aerospace Engineering Sciences and Center for Aerospace \\ Structures, University of Colorado at Boulder, Campus Box 429, Boulder, CO \\ 80309-0429
}

\begin{abstract}
The FETI-DP domain decomposition method (DDM) is extended to address the iterative solution of a class of indefinite problems of the form $(\mathbf{A}-\sigma \mathbf{M}) \mathbf{x}=\mathbf{b}$, where $\mathbf{A}$ and $\mathbf{M}$ are two real symmetric positive semi-definite matrices arising from the finite element discretization of second-order elastodynamic problems, and $\sigma$ is a positive number. A key component of this extension is a new coarse problem based on the free-space solutions of Navier's homogeneous displacement equations of motion. These solutions are waves, and therefore the resulting DDM is reminiscent of the FETI-H method. For this reason, it is named here the FETI-DPH method. For a given $\sigma$, this method is numerically shown to be scalable with respect to all of the problem size, subdomain size, and number of subdomains. Its intrinsic CPU performance is illustrated for various ranges of $\sigma$ with the solution on an Origin 3800 parallel processor of several large-scale structural dynamics problems.
\end{abstract}

Key words: domain decomposition, FETI-DP, FETI-H, GMRES, FETI-DPH, elastodynamics, elastic waves, impedance, shifted matrix, indefinite systems.

* Corresponding author.

Email addresses: charbel.farhat@colorado.edu (Charbel Farhat), jingli@colorado.edu (Jing Li). 


\section{Introduction}

Real linear or linearized systems of equations of the form

$$
(\mathbf{A}-\sigma \mathbf{M}) \mathbf{x}=\mathbf{b}
$$

are frequent in computational structural dynamics. They are encountered, for example, in the finite element simulation of the forced response of a secondorder mechanical system to a periodic excitation [1]. In this case, $\mathbf{A}$ and $\mathbf{M}$ are the finite element stiffness and mass matrices of the considered mechanical system, respectively, $\sigma$ is the square of the circular frequency of the external periodic excitation, $\mathbf{b}$ is its amplitude, $(\mathbf{A}-\sigma \mathbf{M})$ is the impedance of the mechanical system, and $\mathbf{x}$ is the amplitude of its forced response. Such problems also arise during the solution by an inverse shifted method [2] of the generalized symmetric eigenvalue problem $\mathbf{A x}=\omega^{2} \mathbf{M x}$ associated with a second-order mechanical system. In this example, $\mathbf{A}$ and $\mathbf{M}$ have the same meaning as in the previous case, $\omega^{2}$ and $\mathbf{x}$ are a sought-after pair of eigenvalue and eigenvector representing the square of a natural circular frequency and the corresponding natural vibration mode of the mechanical system, respectively, and the shift $\sigma$ is introduced to obtain quickly the closest eigenvalues to $\sigma$. In both examples mentioned here, the matrices $\mathbf{A}$ and $\mathbf{M}$ are usually symmetric positive semi-definite, and therefore $(\mathbf{A}-\sigma \mathbf{M})$ rapidly becomes indefinite when $\sigma$ is increased.

With the pressing need for higher-fidelity three-dimensional structural models with millions of degrees of freedom (dof), and the extreme demands placed by direct methods on computer resources for solving the associated large-scale linear and linearized systems of equations, a large segment of the computational structural mechanics community has shifted its attention from direct to iterative solution strategies. More specifically, domain decomposition (DD) based preconditioned conjugate gradient (PCG) methods have emerged as powerful equation solvers in this field on both sequential and parallel computing platforms [3]. However, most if not all successful domain decomposition methods (DDMs) have been designed for the solution of symmetric positive (semi) definite systems, and typically fail or underperform when applied to the solution of problem (1) with a relatively large $\sigma$. The objective of this paper is to fill this gap in the range of applications of DDMs by extending the numerically scalable FETI-DP [4,5] DDM to the solution of problem (1), when A and $\mathbf{M}$ result from the finite element discretization of the three-dimensional secondorder elastodynamic equations. For this purpose, the reminder of this paper is organized as follows.

In Section 2, the FETI-DP method is overviewed and its scalability properties are summarized. In Section 3, the relationship between problem (1) and Navier's displacement equations of motion is recalled. This relation is exploited 
to construct an auxiliary coarse problem for accelerating the convergence of the solution of problem (1) by a FETI-DP type method named here the FETIDPH method. In Section 4, a preliminary investigation of the performance and scalability properties of the FETI-DPH method is performed for large-scale structural dynamics problems. Section 5 concludes this paper.

\section{The FETI-DP method}

The finite element tearing and interconnecting (FETI) methods [6-8] are a family of dual DDMs with Lagrange multipliers and auxiliary coarse problems. They were developed during the last decade for the fast parallel (as well as sequential) iterative solution of large-scale systems of equations arising from the finite element discretization of second- [9] and fourth-order [10,11] partial differential equations. Essentially, a FETI method can be viewed as a two-step PCG algorithm where subdomain problems with Dirichlet boundary conditions are solved in the preconditioning step, and related subdomain problems with Neumann boundary conditions are solved in a second step. When a FETI method is equipped with its appropriate preconditioner and auxiliary coarse problem, the condition number $\kappa$ of its interface problem grows asymptotically as $[12-14]$

$$
\kappa=\mathcal{O}\left(1+\log ^{m} \frac{H}{h}\right), \quad m \leq 2,
$$

where $H$ and $h$ denote the subdomain and mesh sizes, respectively. The conditioning result (2) proves the numerical scalability of the FETI methodology with respect to all of the problem size, the subdomain size, and the number of subdomains. Most importantly, this result suggests that a FETI method can be expected to solve large-scale problems in a similar number of iterations as small-scale ones. This in turn suggests that a FETI method that is wellimplemented on a parallel processor should be capable of solving an $n$-times larger problem using an $n$-times larger number of processors in almost a constant amount of CPU time. Indeed, these numerical and parallel scalability properties have been demonstrated in practice for many complex structural mechanics and structural dynamics problems (for example, see $[15,16]$ and the references cited therein).

Most recently, the basic principles governing the design of a FETI method were revisited in order to unify the FETI method for second-order systems [6-9], the two-level FETI method for fourth-order systems [10,11], as well as the various FETI methods developed for the solution of various time-dependent problems $[17,11]$ into a single dual-primal DDM named the FETI-DP method $[4,5]$. This most recent FETI method enjoys the same quasi-optimal condition number estimate (2) for all of second- and fourth-order static and time-dependent 
problems, while employing a more computationally efficient auxiliary coarse problem than the previously developed FETI methods. Its key aspects are summarized next in the context of a generic symmetric positive semi-definite problem of the form

$$
\mathrm{Ax}=\mathrm{b}
$$

where $\mathbf{A}$ has the same meaning as in problem (1), and $\mathbf{b}$ is any right hand-side vector.

\subsection{Domain decomposition and nomenclature}

Let $\Omega$ denote the computational support of a second- or fourth-order elastic or elastodynamic problem whose discretization leads to problem $(3),\left\{\Omega^{(s)}\right\}_{s=1}^{N_{s}}$ denote its decomposition into $N_{s}$ subdomains with matching interfaces $\Gamma^{(s, q)}=$ $\partial \Omega^{(s)} \cap \partial \Omega^{(q)}$, and let $\Gamma=\bigcup_{s=1, q>s}^{s=N_{s}} \Gamma^{(s, q)}$ denote the global interface of this decomposition. Let also $\mathbf{A}^{(s)}, \mathbf{x}^{(s)}$, and $\mathbf{b}^{(s)}$ denote the restrictions to subdomain $\Omega^{(s)}$ of $\mathbf{A}, \mathbf{x}$, and $\mathbf{b}$, respectively.

Let $N_{c}$ of the $N_{I}$ nodes lying on the global interface $\Gamma$ be labeled "corner" nodes (see Fig. 1), $\Gamma_{c}$ denote the set of these corner nodes, and let $\Gamma^{\prime}=\Gamma \backslash \Gamma_{c}$. The selection of the corner nodes is briefly discussed in Section 2.3. If in each subdomain $\Omega^{(s)}$ the unknowns are partitioned into global corner dof designated by the subscript $c$, and "remaining" dof designated by the subscript $r, \mathbf{A}^{(s)}$, $\mathbf{x}^{(s)}$ and $\mathbf{b}^{(s)}$ can be partitioned as follows

$$
\mathbf{A}^{(s)}=\left[\begin{array}{cc}
\mathbf{A}_{r r}^{(s)} & \mathbf{A}_{r c}^{(s)} \\
\mathbf{A}_{r c}^{(s)^{T}} & \mathbf{A}_{c c}^{(s)}
\end{array}\right], \quad \mathbf{x}^{(s)}=\left[\begin{array}{c}
\mathbf{x}_{r}^{(s)} \\
\mathbf{x}_{c}^{(s)}
\end{array}\right] \quad \text { and } \quad \mathbf{b}^{(s)}=\left[\begin{array}{c}
\mathbf{b}_{r}^{(s)} \\
\mathbf{b}_{c}^{(s)}
\end{array}\right]
$$

Introducing the subscript $i$ to designate those $r$-type dof lying in the "interior" of a subdomain, and the subscript $b$ to designate those $r$-type dof located at the interface "boundary" of a subdomain, $\mathbf{x}_{r}^{(s)}$ and $\mathbf{b}_{r}^{(s)}$ can be partitioned as follows

$$
\mathbf{x}_{r}^{(s)}=\left[\begin{array}{c}
\mathbf{x}_{i}^{(s)} \\
\mathbf{x}_{b}^{(s)}
\end{array}\right] \quad \text { and } \quad \mathbf{b}_{r}^{(s)}=\left[\begin{array}{l}
\mathbf{b}_{i}^{(s)} \\
\mathbf{b}_{b}^{(s)}
\end{array}\right]
$$

Let $\mathbf{x}_{c}$ denote the global vector of corner dof, and $\mathbf{x}_{c}^{(s)}$ denote its restriction to $\Omega^{(s)}$. Let also $\mathbf{B}_{r}^{(s)}$ and $\mathbf{B}_{c}^{(s)}$ be the two subdomain Boolean matrices defined by

$$
\mathbf{B}_{r}^{(s)} \mathbf{x}_{r}^{(s)}= \pm \mathbf{x}_{b}^{(s)} \quad \text { and } \quad \mathbf{B}_{c}^{(s)} \mathbf{x}_{c}=\mathbf{x}_{c}^{(s)}
$$

where the \pm sign is set by any convention that implies that $\sum_{s=1}^{N_{s}} \mathbf{B}_{r}^{(s)} \mathbf{x}_{r}^{(s)}$ represents the jump of the solution $\mathbf{x}$ across the subdomain interfaces. Finally, 


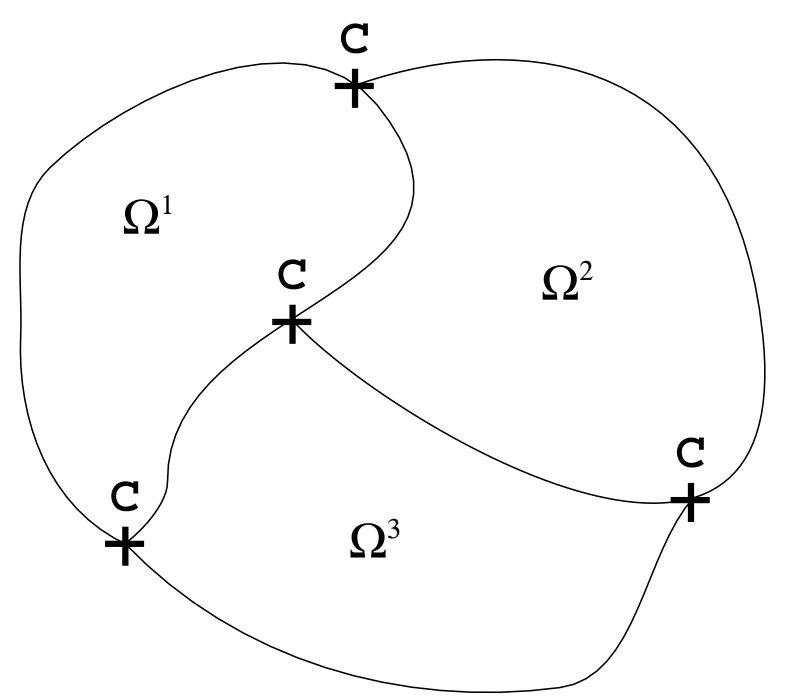

Fig. 1. Example of a definition of corner points.

let

$$
\mathbf{b}_{c}=\sum_{s=1}^{N_{s}} \mathbf{B}_{c}^{(s)^{T}} \mathbf{b}_{c}^{(s)} .
$$

In $[4,5]$, it was shown that solving problem (3) is equivalent to solving the following domain-decomposed problem

$$
\begin{aligned}
\mathbf{A}_{r r}^{(s)} \mathbf{x}_{r}^{(s)}+\mathbf{A}_{r c}^{(s)} \mathbf{B}_{c}^{(s)} \mathbf{x}_{c}+\mathbf{B}_{r}^{(s)^{T}} \lambda+\mathbf{B}_{r}^{(s)^{T}} \mathbf{Q}_{b} \mu & =\mathbf{b}_{r}^{(s)}, \quad s=1, \ldots, N_{s} \\
\sum_{s=1}^{N_{s}} \mathbf{B}_{c}^{(s)^{T}} \mathbf{A}_{r c}^{(s)^{T}} \mathbf{x}_{r}^{(s)}+\sum_{s=1}^{N_{s}} \mathbf{B}_{c}^{(s)^{T}} \mathbf{A}_{c c}^{(s)} \mathbf{B}_{c}^{(s)} \mathbf{x}_{c} & =\mathbf{b}_{c} \\
\sum_{s=1}^{N_{s}} \mathbf{B}_{r}^{(s)} \mathbf{x}_{r}^{(s)} & =0 \\
\mathbf{Q}_{b}^{T} \sum_{s=1}^{N_{s}} \mathbf{B}_{r}^{(s)} \mathbf{x}_{r}^{(s)} & =0
\end{aligned}
$$

where the superscript $T$ designates the transpose, $\lambda$ is an $N_{\lambda}$-long vector of Lagrange multipliers introduced on $\Gamma^{\prime}$ to enforce the continuity (10) of the solution $\mathbf{x}$, and $\mu$ is another vector of Lagrange multipliers introduced to enforce the optional linear constraint (11). This optional constraint, whose concept was developed in [18], is determined by a matrix $\mathbf{Q}_{b}$ with $N_{Q}<N_{\lambda}$ columns defined on $\Gamma^{\prime}$. The word "optional" refers to the fact that Eq. (11) and the vector of Lagrange multipliers $\mu$ are not necessarily needed for formulating the above domain-decomposed problem. On the other hand, since the solution of problem (3) is continuous across the subdomain interfaces, it satisfies Eq. (10) and therefore satisfies Eq. (11) for any matrix $\mathbf{Q}_{b}$. Note also that each of Eqs. (8) is a local subdomain equation, whereas Eq. (9) is an assembled global equation. 
The domain-decomposed problem (8-11) was labeled in $[4,5]$ a "dual-primal" problem because it is formulated in terms of two different types of global unknowns: the dual Lagrange multipliers represented by the vector $\lambda$, and the primal corner dof represented by the vector $\mathbf{x}_{c}$.

In the remainder of this paper, the $j$-th column of $\mathbf{Q}_{b}$ is denoted by $\mathbf{q}_{b_{j}}$ so that

$$
\mathbf{Q}_{b}=\left[\begin{array}{lllll}
\mathbf{q}_{b_{1}} & \cdots & \mathbf{q}_{b_{j}} & \cdots & \mathbf{q}_{b_{N_{Q}}}
\end{array}\right] .
$$

\subsection{Interface and coarse problems}

Let

$$
\begin{aligned}
\widetilde{\mathbf{A}}_{c c}=\left[\begin{array}{rr}
\mathbf{A}_{c c} & 0 \\
0 & 0
\end{array}\right], & \mathbf{d}_{r}=\sum_{s=1}^{N_{s}} \mathbf{B}_{r}^{(s)} \mathbf{A}_{r r}^{(s)^{-1}} \mathbf{b}_{r}^{(s)}, \\
\text { and } & \mathbf{b}_{c}^{*}=\mathbf{b}_{c}-\sum_{s=1}^{N_{s}}\left(\mathbf{A}_{r c}^{(s)} \mathbf{B}_{c}^{(s)}\right)^{T} \mathbf{A}_{r r}^{(s)^{-1}} \mathbf{b}_{r}^{(s)} .
\end{aligned}
$$

After some algebraic manipulations aimed at eliminating symbolically $\mathbf{x}_{r}^{(s)}$, $s=1, \ldots, N_{s}, \mathbf{x}_{c}$, and $\mu$, the domain-decomposed problem (8-11) can be transformed into the following symmetric positive semi-definite interface problem

$$
\left(\mathbf{F}_{I_{r r}}+\widetilde{\mathbf{F}}_{I_{r c}} \widetilde{\mathbf{A}}_{c c}^{*^{-1}} \widetilde{\mathbf{F}}_{I_{r c}}^{T}\right) \lambda=\mathbf{d}_{r}-\widetilde{\mathbf{F}}_{I_{r c}} \widetilde{\mathbf{A}}_{c c}^{*^{-1}} \tilde{\mathbf{b}}_{c}^{*}
$$

where

$$
\begin{aligned}
\mathbf{F}_{I_{r r}}= & \sum_{s=1}^{N_{s}} \mathbf{B}_{r}^{(s)} \mathbf{A}_{r r}^{(s)^{-1}} \mathbf{B}_{r}^{(s)^{T}}, \quad \widetilde{\mathbf{F}}_{I_{r c}}=\sum_{s=1}^{N_{s}} \mathbf{B}_{r}^{(s)} \mathbf{A}_{r r}^{(s)^{-1}} \widetilde{\mathbf{A}}_{r c}^{(s)}, \\
\widetilde{\mathbf{A}}_{r c}^{(s)}= & {\left[\begin{array}{ll}
\mathbf{A}_{r c}^{(s)} \mathbf{B}_{c}^{(s)} & \mathbf{B}_{r}^{(s)^{T}} \mathbf{Q}_{b}
\end{array}\right], } \\
\widetilde{\mathbf{A}}_{c c}^{*}= & \widetilde{\mathbf{A}}_{c c}- \\
& \sum_{s=1}^{N_{s}}\left[\begin{array}{ll}
\left(\mathbf{A}_{r c}^{(s)} \mathbf{B}_{c}^{(s)}\right)^{T} \mathbf{A}_{r r}^{(s)^{-1}}\left(\mathbf{A}_{r c}^{(s)} \mathbf{B}_{c}^{(s)}\right) & \left.\left(\mathbf{A}_{r c}^{(s)} \mathbf{B}_{c}^{(s)}\right)^{T} \mathbf{A}_{r r}^{(s)}\right)^{-1}\left(\mathbf{B}_{r}^{(s)^{T}} \mathbf{Q}_{b}\right) \\
\left(\mathbf{B}_{r}^{(s)^{T}} \mathbf{Q}_{b}\right)^{T} \mathbf{A}_{r r}^{(s)^{-1}}\left(\mathbf{A}_{r c}^{(s)} \mathbf{B}_{c}^{(s)}\right) & \left(\mathbf{B}_{r}^{(s)^{T}} \mathbf{Q}_{b}\right)^{T} \mathbf{A}_{r r}^{(s)}\left(\mathbf{B}_{r}^{(s)^{T}} \mathbf{Q}_{b}\right)
\end{array}\right], \\
\tilde{\mathbf{b}}_{c}^{*}= & {\left[\begin{array}{c}
\mathbf{b}_{c}^{*} \\
-\mathbf{Q}_{b}^{T} \mathbf{d}_{r}
\end{array}\right] . }
\end{aligned}
$$

The FETI-DP method is a DDM which solves the original problem (3) by applying a PCG algorithm to the solution of the corresponding dual inter- 
face problem (14). At each $n$-th PCG iteration, the matrix-vector product $\left(\mathbf{F}_{I_{r r}}+\widetilde{\mathbf{F}}_{I_{r c}} \widetilde{\mathbf{A}}_{c c}^{*^{-1}} \widetilde{\mathbf{F}}_{I_{r c}}^{T}\right) \lambda^{n}$ incurs the solution of an auxiliary problem of the form

$$
\widetilde{\mathbf{A}}_{c c}^{*} \mathbf{z}=\widetilde{\mathbf{F}}_{I_{r c}}^{T} \lambda^{n} .
$$

From the fourth of Eqs. (15), it follows that the size of this auxiliary problem is equal to the sum of the number of corner dof, $N_{c}^{\text {dof }}$, and the number of columns of the arbitrary matrix $\mathbf{Q}_{b}, N_{Q}$.

For $N_{Q}=0$ - that is, for $\mathbf{Q}_{b}=0$, the auxiliary problem (16) is a coarse problem, and $\widetilde{\mathbf{A}}_{c c}^{*}$ is a sparse matrix whose pattern is that of the stiffness matrix obtained when each subdomain is treated as a "superelement" whose nodes are its corner nodes. This coarse problem ensures that the FETI-DP method equipped with the Dirichlet preconditioner (see Section 2.4) is numerically scalable for fourth-order and two-dimensional second-order problems $[5,19]$. However, for $\mathbf{Q}_{b}=0$, the FETI-DP method equipped with the Dirichlet preconditioner is not numerically scalable for three-dimensional second-order problems.

For any choice of $\mathbf{Q}_{b} \neq 0, \widetilde{\mathbf{A}}_{c c}^{*}$ remains a sparse matrix. If $\mathbf{Q}_{b}$ is constructed edge-wise - that is, if each column of $\mathbf{Q}_{b}$ is constructed as the restriction of some operator to a specific edge of $\Gamma^{\prime}$ - the sparsity pattern of $\widetilde{\mathbf{A}}_{c c}^{*}$ becomes that of a stiffness matrix obtained by treating each subdomain as a superelement whose nodes are its corner nodes augmented by virtual mid-side nodes. The number of dof attached to each virtual mid-side node is equal to the number of columns of $\mathbf{Q}_{b}$ associated with the edge on which lies this midside node. If $N_{Q}$ is kept relatively small, the auxiliary problem (16) remains a relatively small coarse problem. This coarse problem was labeled in [4] the "augmented" coarse problem in order to distinguish it from the smaller coarse problem obtained with $\mathbf{Q}_{b}=0$. Furthermore, each column of $\mathbf{Q}_{b}$ is referred to as an "augmentation coarse mode". When for three-dimensional problems these augmentation coarse modes are chosen as the translational rigid body modes of each edge of $\Gamma^{\prime}$ — that is, when each three consecutive columns of $\mathbf{Q}_{b}$ are constructed as follows

$$
\begin{aligned}
& \mathbf{q}_{b_{x}}=\left[\begin{array}{lllllllllllllllll}
0 & \cdots & 0 & {\left[\begin{array}{lllllllllll}
1 & 0 & 0 & \cdots & 1 & 0 & 0 & \cdots & 1 & 0 & 0
\end{array}\right]} & \cdots & 0
\end{array}\right]^{T}, \\
& \mathbf{q}_{b_{y}}=\left[\begin{array}{lllllllllllllllll}
0 & \cdots & 0 & {\left[\begin{array}{llllllllllll}
0 & 1 & 0 & \cdots & 0 & 1 & 0 & \cdots & 0 & 1 & 0
\end{array}\right]} & \cdots & \cdots & 0
\end{array}\right]^{T}, \\
& \left.\mathbf{q}_{b_{z}}=\left[\begin{array}{lllllllllllllllll}
0 & \cdots & 0 & 0 & 0 & 1 & \cdots & 0 & 0 & 1 & \cdots & 0 & 0 & 1
\end{array}\right] \quad \begin{array}{llll}
0 & \cdots
\end{array}\right]^{T} \text {, }
\end{aligned}
$$

the FETI-DP method equipped with the Dirichlet preconditioner becomes numerically scalable for three-dimensional second-order problems [20]. In Eqs. (17) above, a block of the form 100 refers to the displacement at an interface node induced by a rigid body mode in the $x$ direction, a block of the form 010 refers to the displacement at an interface node induced by a rigid body mode 
in the $y$ direction, and a block of the form 001 refers to the displacement at an interface node induced by a rigid body mode in the $z$ direction.

\subsection{Corner selection}

From the definitions of $\mathbf{F}_{I_{r r}}, \widetilde{\mathbf{A}}_{c c}^{*}$, and $\mathbf{d}_{r}$ given in $(15,13)$, it follows that the corner nodes must be chosen so that $\mathbf{A}_{r r}^{(s)}$ is non-singular. From Eq. (14), it follows that when $\mathbf{Q}_{b} \neq 0$, the selection of the corner nodes must futhermore guarantee that $\widetilde{\mathbf{A}}_{c c}^{*}$ is non-singular. A corner selection algorithm that meets both of these requirements was proposed and discussed in [21].

\section{$2.4 \quad$ Preconditioning}

Two preconditioners have been developed so far for the FETI-DP method:

(1) The Dirichlet preconditioner [9] which can be written as

$$
\begin{aligned}
\overline{\mathbf{F}}_{I_{r r}}^{D^{-1}} & =\sum_{s=1}^{N_{s}} \mathbf{W}^{(s)} \mathbf{B}_{r}^{(s)}\left[\begin{array}{cc}
0 & 0 \\
0 & \mathbf{S}_{b b}^{(s)}
\end{array}\right] \mathbf{B}_{r}^{(s)^{T}} \mathbf{W}^{(s)}, \\
\text { where } \quad \mathbf{S}_{b b}^{(s)} & =\mathbf{A}_{b b}^{(s)}-\mathbf{A}_{i b}^{(s)^{T}} \mathbf{A}_{i i}^{(s)^{-1}} \mathbf{A}_{i b}^{(s)},
\end{aligned}
$$

the subscripts $i$ and $b$ have the same meaning as in Section 2.1, and $\mathbf{W}^{(s)}$ is a subdomain diagonal scaling matrix that accounts for eventual subdomain heterogeneities [22]. This preconditioner is mathematically optimal in the sense that it leads to the condition number estimate (2).

(2) The lumped preconditioner [7] which can be written as

$$
\overline{\mathbf{F}}_{I_{r r}}^{L^{-1}}=\sum_{s=1}^{N_{s}} \mathbf{W}^{(s)} \mathbf{B}_{r}^{(s)}\left[\begin{array}{cc}
0 & 0 \\
0 & \mathbf{A}_{b b}^{(s)}
\end{array}\right] \mathbf{B}_{r}^{(s)^{T}} \mathbf{W}^{(s)}
$$

This preconditioner is not mathematically optimal.

The Dirichlet preconditioner is more computationally expensive than the lumped preconditioner, but is also more computationally efficient for fourth-order problems. However, for second-order problems, the lumped preconditioner is more computationally efficient than the Dirichlet preconditioner, even though it is not mathematically optimal. 


\section{The FETI-DPH method}

In the context of problem (1), $\mathbf{A}_{r r}^{(s)}$ becomes $\mathbf{A}_{r r}^{(s)}-\sigma \mathbf{M}_{r r}^{(s)}$. Hence, the extension of the FETI-DP method to problems of the form given in (1) requires addressing the following issues:

(1) $\mathbf{A}_{r r}^{(s)}-\sigma \mathbf{M}_{r r}^{(s)}$ is indefinite and therefore the dual interface problem (14) is indefinite.

(2) Independently of which interface points are chosen to be corner points, $\mathbf{A}_{r r}^{(s)}-\sigma \mathbf{M}_{r r}^{(s)}$ is in theory singular when $\sigma$ happens to be an eigenvalue of the pencil $\left(\mathbf{A}_{r r}^{(s)}, \mathbf{M}_{r r}^{(s)}\right)$.

(3) How to choose the augmentation coarse modes and use them to construct the matrix $\mathbf{Q}_{b}$.

Here, the first issue is addressed by proposing to solve the dual interface problem (14) by a preconditioned generalized minimum residual (PGMRES) algorithm [23] rather than a PCG algorithm.

The second and third issues were addressed in [24] in the context of the basic FETI method and acoustic scattering problems - that is, for the case of the exterior scalar Helmholtz equation where $\sigma=k^{2}$ and $k$ denotes the wave number. More specifically, a regularization procedure was developed in [24] to prevent all subdomain problems from being singular for any value of the wave number $k$, without destroying the sparsity of the local matrices $\mathbf{A}_{r r}^{(s)}-k^{2} \mathbf{M}_{r r}^{(s)}$ and without affecting the solution of the original problem (1). Furthermore, for the scalar Helmholtz equation, the coarse modes were chosen in [24] as vectors of plane waves of the form $e^{i k \theta_{j}^{T} X_{b}}, j=1,2, \cdots$, where $\theta_{j}$ denotes a direction of wave propagation and $X_{b}$ the coordinates of a node on $\Gamma$. The resulting DDM was called in [24] the FETI-H method ( $\mathrm{H}$ for Helmholtz).

Unfortunately, the regularization procedure characterizing the FETI-H method transforms each real subdomain problem into a complex subdomain problem. For acoustic scattering problems, this incurs a minor computational overhead because in this case, the Sommerfeld radiation condition causes the undecomposed algebraic problem (1) to be in the complex domain. However, when this problem is originally formulated in the real domain, the regularization procedure of the FETI-H method becomes unjustifiable from a computational performance viewpoint.

In practice, experience reveals that perhaps because of round-off effects, for any value of $\sigma, \mathbf{A}_{r r}^{(s)}-\sigma \mathbf{M}_{r r}^{(s)}$ is never singular as long as $\mathbf{A}_{r r}^{(s)}$ is non-singular. This observation is exploited here to design an extension of the FETI-DP method for indefinite problems of the form given in (1) by:

(1) Replacing the PCG solver by the PGMRES solver. 
(2) Keeping the same Dirichlet and lumped preconditioners but replacing in (18) and (19) $\mathbf{A}_{l m}^{(s)}$ by $\mathbf{A}_{l m}^{(s)}-\sigma \mathbf{M}_{l m}^{(s)}, l=i, b$, and $m=i, b$.

(3) Choosing the augmentation coarse space and constructing its representative matrix $\mathbf{Q}_{b}$ as described next.

\subsection{Edge-based elastic wave modes}

Let $\mathbf{r}$ denote the residual associated with the iterative solution of the dual interface problem (14). From Eqs. (8-11) and Eq. (14), it follows that

$$
\mathbf{r}=\mathbf{d}_{r}-\widetilde{\mathbf{F}}_{I_{r c}} \widetilde{\mathbf{A}}_{c c}^{*-1} \tilde{\mathbf{b}}_{c}^{*}-\left(\mathbf{F}_{I_{r r}}+\widetilde{\mathbf{F}}_{I_{r c}} \widetilde{\mathbf{A}}_{c c}^{*^{-1}} \widetilde{\mathbf{F}}_{I_{r c}}^{T}\right) \lambda=\sum_{s=1}^{N_{s}} \mathbf{B}_{r}^{(s)} \mathbf{x}_{r}^{(s)},
$$

which reveals that the residual $\mathbf{r}$ represents the jump of the iterate solution across the subdomain interfaces. Hence, the FETI-DP method converges when the iterate solution $\mathrm{x}^{n}$ becomes continuous across all the subdomain interfaces.

From Eq. (11), Eq. (15), Eq. (14) and Eq. (10), it follows that at each iteration of the PGMRES algorithm applied to the solution of problem (14), FETIDP forces the jump of the solution across the subdomain interfaces to be orthogonal to the subspace represented by the matrix $\mathbf{Q}_{b}$. This feature is a strategy for designing an auxiliary coarse problem which, when $\mathbf{Q}_{b}$ is well chosen, accelerates the convergence of a DDM [18].

In this work, the search for a suitable matrix $\mathbf{Q}_{b}$ is driven by the following reasoning. Suppose that the space of traces on $\Gamma^{\prime}$ of the sought-after solution of problem (1) is spanned by a set of orthogonal vectors $\left\{\mathbf{v}_{j_{E}}\right\}_{j=1}^{N_{\lambda}}$, where the subscript $E$ indicates that $\mathbf{v}_{j_{E}}$ is non-zero only on edge $E \in \Gamma^{\prime}$. Then, the residual $\mathbf{r}$ defined in Eq. (20) can be written as

$$
\mathbf{r}=\sum_{j=1}^{N_{\lambda}} \alpha_{j} \mathbf{v}_{j_{E}}
$$

where $\left\{\alpha_{j}\right\}_{j=1}^{N_{\lambda}}$ is a set of real coefficients. If each augmentation coarse mode is chosen as

$$
\mathbf{q}_{b_{j}}=\mathbf{v}_{j_{E}}, \quad j=1, \cdots, N_{Q}
$$

Eq. (11) simplifies to

$$
\alpha_{j}=0, \quad j=1, \cdots, N_{Q} .
$$

In this case, Eq. (23) implies that at each iteration of the PGMRES algorithm, the first $N_{Q}$ components of the residual $\mathbf{r}$ in the basis $\left\{\mathbf{v}_{j_{E}}\right\}_{j=1}^{N_{\lambda}}$ are zero. If a few vectors $\left\{\mathbf{v}_{j_{E}}\right\}_{j=1}^{N_{Q}}, N_{Q}<<N_{\lambda}$, that dominate the expansion (21) can 
be found, then choosing these vectors as coarse augmentation modes can be expected to accelerate the convergence of the iterative solution of the dual interface problem (14). Hence, it remains to exhibit such a set of orthogonal vectors $\mathbf{v}_{j_{E}}$ and construct a computationally efficient matrix $\mathbf{Q}_{b}$.

As stated in Section 1, the focus of this paper is on structural dynamics applications where $\mathbf{A}$ and $\mathbf{M}$ typically arise from the finite element discretization of a three-dimensional elastodynamic or elastodynamic-like problem. Such a problem is governed by Navier's displacement equations of motion

$$
\mu \Delta u+(\Lambda+\mu) \nabla(\nabla \cdot u)+b=\rho \frac{\partial^{2} u}{\partial t^{2}}
$$

where $u \in \mathbb{R}^{3}$ denotes the displacement (vector) field of the elastodynamic system, $\Lambda$ and $\mu$ its Lamé moduli, $b \in \mathbb{R}^{3}$ its body forces, $\rho$ its density, and $t$ denotes time. If a harmonic motion is assumed, - that is, if

$$
u(X, t)=v(X) e^{-i \omega t},
$$

where $i$ denotes here the pure imaginary number satisfying $i^{2}=-1, X \in \mathbb{R}^{3}$ denotes the spatial variables, and $\omega$ denotes a circular frequency, the homogeneous form of Eq. (24) becomes

$$
\mu \Delta v+(\Lambda+\mu) \nabla(\nabla \cdot v)+\rho \omega^{2} v=0 .
$$

The free-space solutions of the above vector equation are

$$
\begin{array}{ll}
v=a_{p} \sin \left(k_{p} \theta \cdot X\right), & v=a_{p} \cos \left(k_{p} \theta \cdot X\right), \\
v=a_{s_{1}} \sin \left(k_{s} \theta \cdot X\right), & v=a_{s_{1}} \cos \left(k_{s} \theta \cdot X\right), \\
v=a_{s_{2}} \sin \left(k_{s} \theta \cdot X\right), & v=a_{s_{2}} \cos \left(k_{s} \theta \cdot X\right),
\end{array}
$$

where $\theta \in \mathbb{R}^{3}$ is an arbitrary vector of unit length $\left(\|\theta\|_{2}=1\right), a_{p} \in \mathbb{R}^{3}$ is a vector that is parallel to $\theta,\left(a_{s_{1}}, a_{s_{2}}\right) \in \mathbb{R}^{3} \times \mathbb{R}^{3}$ are two independent vectors in the plane orthogonal to $\theta$,

$$
k_{p}=\sqrt{\frac{\rho \omega^{2}}{\Lambda+2 \mu}}, \quad \text { and } \quad k_{s}=\sqrt{\frac{\rho \omega^{2}}{\mu}} \text {. }
$$

The free-space solutions (27) are known as the elastic pressure or longitudinal waves, and the free-space solutions (28) and (29) are known as the elastic shear or transverse waves.

Hence, the general solution of Eq. (26) can be written as 


$$
\begin{aligned}
v & =\sum_{j=1}^{\infty}\left\{a_{p_{j}}\left(\beta_{j} \sin \left(k_{p} \theta_{j} \cdot X\right)+\gamma_{j} \cos \left(k_{p} \theta_{j} \cdot X\right)\right)\right\} \\
& +\sum_{j=1}^{\infty}\left\{a_{s_{1 j}}\left(\delta_{j} \sin \left(k_{s} \theta_{j} \cdot X\right)+\zeta_{j} \cos \left(k_{s} \theta_{j} \cdot X\right)\right)\right\} \\
& +\sum_{j=1}^{\infty}\left\{a_{s_{2 j}}\left(\eta_{j} \sin \left(k_{s} \theta_{j} \cdot X\right)+\xi_{j} \cos \left(k_{s} \theta_{j} \cdot X\right)\right)\right\},
\end{aligned}
$$

where $\theta_{j} \in \mathbb{R}^{3}$ is an arbitrary vector of unit length defining the direction of propagation of an elastic pressure or shear wave, and $\beta_{j}, \gamma_{j}, \delta_{j}, \zeta_{j}, \eta_{j}$, and $\xi_{j}$ are real coefficients. From Eq. (31) and Eq. (22), it follows that the soughtafter matrix $\mathbf{Q}_{b}$ is composed of blocks of six columns. The six columns $\mathbf{q}_{b_{l+q}}$, $q=0,1, \ldots, 5$, of each block are associated with one direction of propagation $\theta_{j}$ and one edge $E$ of the mesh partition, and can be written as

$$
\mathbf{q}_{b_{l+q}}\left[\begin{array}{c}
3(m-1)+1 \\
3(m-1)+2 \\
3(m-1)+3
\end{array}\right]= \begin{cases}a_{p_{j}} \sin \left(k_{p} \theta_{j} \cdot X_{m}\right), & q=0, \\
a_{p_{j}} \cos \left(k_{p} \theta_{j} \cdot X_{m}\right), & q=1, \\
a_{s_{1 j}} \sin \left(k_{s} \theta_{j} \cdot X_{m}\right), & q=2, \\
a_{s_{1 j}} \cos \left(k_{s} \theta_{j} \cdot X_{m}\right), & q=3, \\
a_{s_{2 j}} \sin \left(k_{s} \theta_{j} \cdot X_{m}\right), & q=4, \\
a_{s_{2 j}} \cos \left(k_{s} \theta_{j} \cdot X_{m}\right), & q=5, \\
l & =6(j-1)+1, \\
m & =1, \cdots, N_{I}-N_{c},\end{cases}
$$

where $\mathbf{q}_{b}[3(m-1)+1]$ designates the entry of $\mathbf{q}_{b}$ associated with the dof in the $x$-direction attached to the $m$-th node on an edge $E \in \Gamma^{\prime}, \mathbf{q}_{b}[3(m-$ $1)+2$ ] designates the entry associated with the dof along the $y$-direction, $\mathbf{q}_{b}[3(m-1)+3]$ designates the entry associated with the dof along the $z$ direction, and $X_{m} \in \mathbb{R}^{3}$ denotes the coordinates of this $m$-th node. Hence, if $N_{E}$ denotes the number of edges of the mesh partition, and $N_{\theta}$ the number of considered directions of wave propagation, the total number of augmentation coarse modes is given in general by $N_{Q}=6 N_{E} N_{\theta}$. To these modes can be added the edge-based translational rigid body modes (17) as these are freespace solutions of Eq. (26) when $\omega=0$.

In this paper, the number of directions is limited by $N_{\theta}^{\max }=13$, and the directions $\theta_{j}$ are generated as follows. A generic cube is discretized into $3 \times 3 \times 3$ points. A direction $\theta_{j}$ is defined by connecting the center point to any of the other 26 points lying on a face of the cube. Since each direction $\theta_{j}$ is used to define both a cosine and a sine mode, only one direction $\theta_{j}$ is retained for each pair of opposite directions, which results in a maximum of 13 directions. 
There are at least three mechanisms that can cause the matrix $\mathbf{Q}_{b}$ described in Section 3.1 to be rank deficient:

(1) If a direction $\theta_{j}$ turns out to be perpendicular to an edge $E \in \Gamma^{\prime}, \theta_{j} \cdot X_{m}$ becomes constant for all $X_{m} \in E$, the rank of the six-column block of $\mathbf{Q}_{b}$ associated with the edge $E$ and the direction $\theta_{j}$ becomes equal to three, and therefore $\mathbf{Q}_{b}$ becomes rank deficient.

(2) Similarly, if a direction $\theta_{j}$ turns out to be perpendicular to all vectors $X_{m} \in E, \theta_{j} \cdot X_{m}=0$, the rank of the six-column block of $\mathbf{Q}_{b}$ associated with $E$ and $\theta_{j}$ becomes equal to three, and therefore $\mathbf{Q}_{b}$ becomes rank deficient.

(3) In Appendix, it is shown that an interplay between a low mesh resolution and $N_{\theta}$ can also cause $\mathbf{Q}_{b}$ to become rank deficient.

From Eq. (11) or the definition of $\widetilde{\mathbf{A}}_{c c}^{*}$ given in the fourth of Eqs. (15), it follows that whenever $\mathbf{Q}_{b}$ does not have full column rank, $\widetilde{\mathbf{A}}_{c c}^{*}$ becomes singular. Singular coarse problems can be solved by a number of different techniques, among which the drop-tolerance based direct method described in [25]. However, preventing $\mathbf{Q}_{b}$ from being rank deficient — and therefore preventing $\widetilde{\mathbf{A}}_{c c}^{*}$ from being singular — is desirable as this improves the computational efficiency as well as the robustness of the solution of the coarse problem (16), and therefore enhances the efficiency and robustness of the overall solution method.

Let $\epsilon_{Q R}$ denote a small tolerance value. The following describes a simple method for filtering the matrix of augmentation coarse modes $\mathbf{Q}_{b}$ and transforming it into a matrix $\mathbf{Q}_{b}^{*}$ that has full column rank:

(1) Perform the QR factorization [26] of $\mathbf{Q}_{b}$.

(2) Construct $\mathbf{Q}_{b}^{*}$ as the union of the columns $\mathbf{q}_{b_{j}}$ of $\mathbf{Q}_{b}$ for which $\mathbf{R}_{j j}>\epsilon_{Q R}$.

The following observations are worthy noting:

(1) Since each column of $\mathbf{Q}_{b}$ has non-zero entries only for the dof associated with a corresponding edge $E$, the $\mathbf{Q R}$ factorization of $\mathbf{Q}_{b}$ entails only local computations that can be performed on an edge-by-edge basis. Hence, these computations are amenable to an efficient parallelization.

(2) A too small value of $\epsilon_{Q R}$ can result in a matrix $\mathbf{Q}_{b}^{*}$ that is still rank deficient, thereby defeating the purpose of the filtering procedure. On the other hand, a too large value of $\epsilon_{Q R}$ can only cause an excessive filtering. Since the columns of $\mathbf{Q}_{b}$ serve the only purpose of defining optional constraints aimed at accelerating the convergence of the iterative DDM (see Eq. (11)), it follows that this DDM is better served by setting $\epsilon_{Q R}$ to a 
value that is sufficiently large to prevent $\mathbf{Q}_{b}^{*}$ from being rank deficient, even if such a tolerance can cause occasionally an excessive filtering of the matrix $\mathbf{Q}_{b}$.

\subsection{A domain decomposition based preconditioned GMRES solver}

The FETI-DPH method is introduced in this paper as the FETI-DP method equipped with the PGMRES rather than PCG algorithm for solving the dual interface problem (14), and with the wave-based matrix of augmentation coarse modes described in Section 3.1.

\section{Performance analysis}

Here, the FETI-DPH method is applied to the solution of various problems of the form (1) associated with the discretization by quadratic tetrahedral elements (10 nodes per element) of a wheel carrier structure (see Fig. 2) fixed at a few of its nodes. In all these problems, the shift is set to $\sigma=\omega^{2}=4 \pi^{2} f^{2}$, where $\omega^{2}$ is the square of a (possibly natural) circular frequency and $f$ is the corresponding frequency in Hz. As stated in the introduction, such problems arise, for example, in the finite element simulation of the forced response of this structure to a periodic excitation characterized by the frequency $f=\omega / 2 \pi$, or during the eigen analysis of this structure by an inverse shifted method. In both cases, the value of $\sigma$ is related to the natural frequencies of the structure that are reported in Table 1 . In order to investigate the performance, potential, and various scalability properties of the FETI-DPH method, various values of $\omega$ (or $f$ ) are considered and three meshes with different resolutions are employed. The coarsest mesh contains 504,375 dof, the intermediate one contains 1,317,123 dof, and the finest mesh contains 2,091,495 dof.

In call cases, the right hand-side of problem (1) is generated by a distributed load, and the FETI-DPH method is equipped with the lumped preconditioner and $\epsilon_{Q R}=10^{-2}$. The relative residual $R E^{n}$ is monitored and convergence is declared when

$$
R E^{n}=\frac{\left\|(\mathbf{A}-\sigma \mathbf{M}) \mathbf{x}^{n}-\mathbf{b}\right\|_{2}}{\|\mathbf{b}\|_{2}} \leq 10^{-6} .
$$

All computations are performed on a Silicon Graphics Origin 3800 system with $40 \mathrm{R} 12000400 \mathrm{MHz}$ processors. This parallel system consists of 5 bricks (or "boxes") of 8 processors each. In each brick, the 8 processors share 8 gigabytes of real memory. The 5 boxes are interconnected by a Myrinet switch. As coded, the FETI-DPH solver relies on threads or OpenMP for parallel computations within a brick and on MPI for interprocessor communication across different 


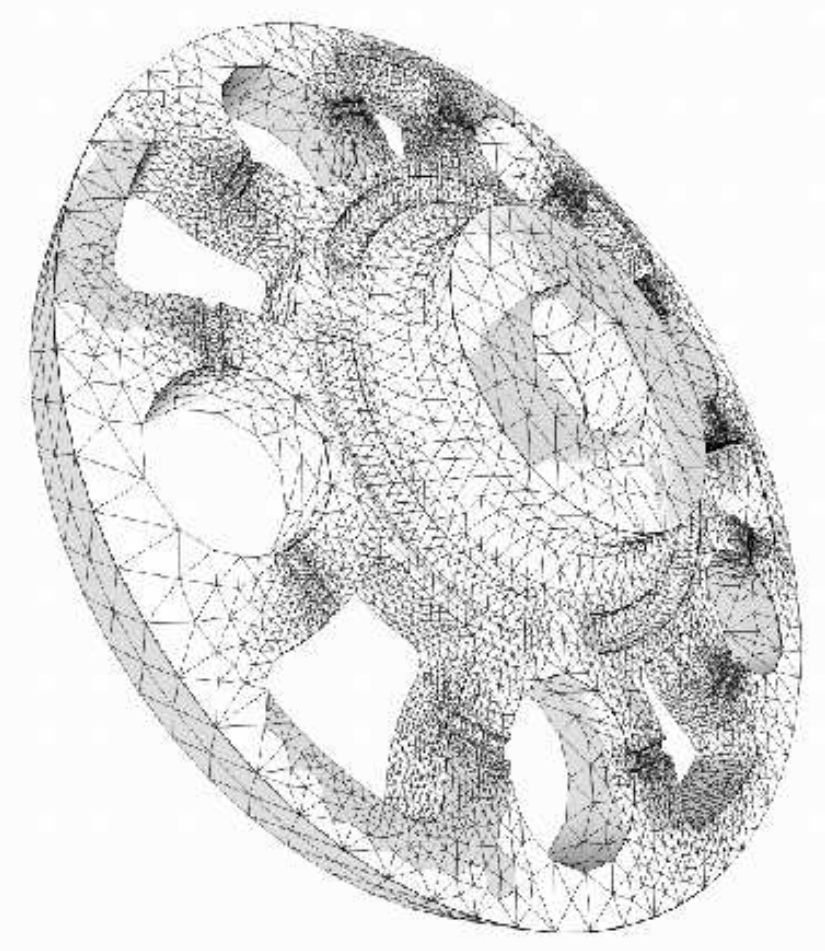

Fig. 2. Finite element discretization of a wheel carrier.

Table 1

Eigenvalue/Frequency partial spectrum of the pencil $(\mathbf{A}, \mathbf{M})$.

\begin{tabular}{||c|c|c||}
\hline \hline Mode Number & Eigenvalue $\left(\omega^{2}\right)$ & Frequency \\
\hline 1 & $2.6 \mathrm{e}+11$ & $8.2 \mathrm{e}+04 \mathrm{~Hz}$ \\
100 & $5.2 \mathrm{e}+13$ & $1.1 \mathrm{e}+06 \mathrm{~Hz}$ \\
200 & $1.6 \mathrm{e}+14$ & $2.0 \mathrm{e}+06 \mathrm{~Hz}$ \\
300 & $2.8 \mathrm{e}+14$ & $2.6 \mathrm{e}+06 \mathrm{~Hz}$ \\
400 & $4.0 \mathrm{e}+14$ & $3.2 \mathrm{e}+06 \mathrm{~Hz}$ \\
500 & $5.1 \mathrm{e}+14$ & $3.5 \mathrm{e}+06 \mathrm{~Hz}$ \\
600 & $6.0 \mathrm{e}+14$ & $3.9 \mathrm{e}+06 \mathrm{~Hz}$ \\
\hline
\end{tabular}

bricks, and does not require that the number of subdomains $N_{s}$ be equal to the number of processors $N_{p}$. 


\subsection{Scalability}

First, attention is directed to the mesh with $N_{\text {dof }}=1,317,123$ which is partitioned into 150, 300,600, and 1,200 subdomains. Four different values of the shift parameter corresponding to $f=10^{5} \mathrm{~Hz}, f=5 \times 10^{5} \mathrm{~Hz}, f=10^{6} \mathrm{~Hz}$, and $f=2 \times 10^{6} \mathrm{~Hz}$ are considered. The last value of the shift parameter corresponds to exciting the structure by its 200-th natural frequency, or shifting around this frequency. In all cases, the matrix $\mathbf{Q}_{b}$ is constructed with $N_{\theta}=2$ as well as the three translational rigid body modes. The performance results of the FETI-DPH solver obtained on a single brick with $N_{p}=8$ processors are reported in Table 2. These results show that:

(1) For each considered value of $\sigma$, the iteration count decreases in general with the number of subdomains, which highlights the numerical scalability of the FETI-DPH method with respect to the number of subdomains.

(2) As can be expected, the iteration count increases when $\sigma$ is increased because problem (1) becomes more indefinite and therefore more difficult to solve iteratively. However, when $\sigma$ is increased by a factor of 500 , the iteration count and CPU time of the FETI-DPH solver are increased by a factor ranging between 2 and 3 only.

Next, all three meshes are considered, and each one is partitioned by a different number of subdomains $N_{s}$ chosen to keep the subdomain problem size constant. Three different frequencies ranging between $500 \mathrm{KHz}$ and $2 \mathrm{MHz}$ are considered, the number of wave directions is set to $N_{\theta}=2$, and the three translational rigid body modes are included in the construction of the augmentation matrix $\mathbf{Q}_{b}$. The performance results of the FETI-DPH solver obtained on $N_{p}=12$ processors are reported in Table 3. For each considered frequency, the iteration count associated with the chosen number of subdomains appears to be almost independent of the mesh size, which highlights the numerical scalability of the FETI-DPH method with respect to both the subdomain problem size and the total problem size. The reader can check that for these computations where the number of subdomains is chosen to maintain constant subdomain problem size, the CPU performance of the FETI-DPH solver scales almost linearly with the problem size.

\subsection{Affordable shifts}

Finally, attention is focused again on the mesh with 1,317,123 dof and the maximum value of the shift parameter is increased to consider the case of a forcing frequency equal to $4 \mathrm{MHz}$. From Table 1, it follows that this frequency is in the neighborhood of the 600-th natural mode and therefore is as high 
Table 2

Performance of the FETI-DPH solver: fixed problem size $N_{\text {dof }}=1,317,123$; increasing number of subdomains $N_{s} ; N_{\theta}=2$ ( + the three translational rigid body modes); $N_{p}=8$.

\begin{tabular}{|c|c|c|c|c|}
\hline Frequency & Shift $\sigma$ & $N_{s}$ & $N_{i t r}$ & $\mathrm{CPU}$ \\
\hline \multirow{4}{*}{$10^{5} \mathrm{~Hz}$} & \multirow{4}{*}{$3.9 \mathrm{e}+11$} & 150 & 78 & $495 \mathrm{~s}$. \\
\hline & & 300 & 72 & $339 \mathrm{~s}$. \\
\hline & & 600 & 62 & $242 \mathrm{~s}$. \\
\hline & & 1,200 & 54 & $291 \mathrm{~s}$. \\
\hline \multirow{4}{*}{$5 \times 10^{5} \mathrm{~Hz}$} & \multirow{4}{*}{$9.8 \mathrm{e}+12$} & 150 & 86 & $540 \mathrm{~s}$. \\
\hline & & 300 & 79 & $371 \mathrm{~s}$. \\
\hline & & 600 & 70 & $280 \mathrm{~s}$. \\
\hline & & 1,200 & 65 & $370 \mathrm{~s}$. \\
\hline \multirow{4}{*}{$10^{6} \mathrm{~Hz}$} & \multirow{4}{*}{$3.9 \mathrm{e}+13$} & 150 & 123 & $682 \mathrm{~s}$. \\
\hline & & 300 & 130 & $529 \mathrm{~s}$. \\
\hline & & 600 & 118 & $428 \mathrm{~s}$. \\
\hline & & 1,200 & 108 & $584 \mathrm{~s}$. \\
\hline \multirow{4}{*}{$2 \times 10^{6} \mathrm{~Hz}$} & \multirow{4}{*}{$1.6 \mathrm{e}+14$} & 150 & 188 & $918 \mathrm{~s}$. \\
\hline & & 300 & 194 & $750 \mathrm{~s}$. \\
\hline & & 600 & 173 & $632 \mathrm{~s}$. \\
\hline & & 1,200 & 160 & $937 \mathrm{~s}$. \\
\hline
\end{tabular}

as imaginable for practical applications. For each considered value of the shift parameter $\sigma$, the number of subdomains $N_{s}$ and number of wave directions $N_{\theta}$ are chosen to maximize the CPU performance of the FETI-DPH solver. This performance is reported in Table 4 together with the performance of the standard FETI-DP solver equipped with the lumped preconditioner, an augmentation matrix $\mathbf{Q}_{b}$ constructed using only the translational rigid body modes, and the PGMRES rather than PCG algorithm for solving the dual interface problem in order to address the indefinite nature of the local subdomain problems. All CPU timings correspond to runs performed on a single brick with $N_{p}=8$ processors. For $\sigma=3.9 e+11$, the standard FETI-DP algorithm is capable of solving problem (1) but is slower than the FETI-DPH solver. For $\sigma=8.9 e+13$, the FETI-DP algorithm almost reaches the stopping criterion (33), but after 8 times more iterations than the FETI-DPH solver and nine times its CPU time. For $\sigma=6.3 e+14$, the FETI-DP solver fails at solving problem (1) whereas the FETI-DPH succeeds at this task with $N_{\theta}=10$. 
Table 3

Performance of the FETI-DPH solver: fixed subdomain problem size; increasing problem size; $N_{\theta}=2$ ( + the three translational rigid body modes) $; N_{p}=12$.

\begin{tabular}{|c|c|c|c|c|c|}
\hline Frequency & Shift $\sigma$ & $N_{d o f}$ & $N_{s}$ & $N_{i t r}$ & $\mathrm{CPU}$ \\
\hline \multirow{3}{*}{$5 \times 10^{5} \mathrm{~Hz}$} & \multirow{3}{*}{$9.8 \mathrm{e}+12$} & 504,375 & 250 & 65 & $64 \mathrm{~s}$. \\
\hline & & $1,317,123$ & 600 & 70 & $215 \mathrm{~s}$. \\
\hline & & $2,091,495$ & 950 & 60 & $368 \mathrm{~s}$. \\
\hline \multirow{3}{*}{$10^{6} \mathrm{~Hz}$} & \multirow{3}{*}{$3.9 \mathrm{e}+13$} & 504,375 & 250 & 116 & $105 \mathrm{~s}$. \\
\hline & & $1,317,123$ & 600 & 118 & $336 \mathrm{~s}$. \\
\hline & & $2,091,495$ & 950 & 122 & $687 \mathrm{~s}$. \\
\hline \multirow{3}{*}{$2 \times 10^{6} \mathrm{~Hz}$} & \multirow{3}{*}{$1.6 \mathrm{e}+14$} & 504,375 & 250 & 137 & $130 \mathrm{~s}$. \\
\hline & & $1,317,123$ & 600 & 173 & $505 \mathrm{~s}$. \\
\hline & & $2,091,495$ & 950 & 151 & $901 \mathrm{~s}$. \\
\hline
\end{tabular}

Table 4

Comparative performances of the FETI-DPH and FETI-DP solvers: $N_{d o f}=$ $1,317,123 ; N_{p}=8$.

\begin{tabular}{||c|c|c|c|c|c|c|}
\hline \hline $\begin{array}{c}\text { Frequency } \\
(\sigma)\end{array}$ & $N_{s}$ & $\begin{array}{c}N_{\theta} \\
\mathrm{DPH}\end{array}$ & $\begin{array}{c}N_{i t r} \\
\mathrm{DPH}\end{array}$ & $\begin{array}{c}N_{i t r} \\
\mathrm{DP}\end{array}$ & $\begin{array}{c}\mathrm{CPU} \\
\mathrm{DPH}\end{array}$ & $\begin{array}{c}\mathrm{CPU} \\
\mathrm{DP}\end{array}$ \\
\hline \hline $\begin{array}{c}10^{5} \mathrm{~Hz} \\
(3.9 \mathrm{e}+11)\end{array}$ & 600 & $2^{*}$ & 62 & 106 & $242 \mathrm{~s}$. & $299 \mathrm{~s}$. \\
\hline $\begin{array}{c}1.5 \times 10^{6} \mathrm{~Hz} \\
(8.9 \mathrm{e}+13)\end{array}$ & 600 & 3 & 115 & $\begin{array}{c}>800 \\
\left(R E^{k}=10^{-5}\right)\end{array}$ & $528 \mathrm{~s}$. & $>4,580 \mathrm{~s}$. \\
\hline $\begin{array}{c}4.0 \times 10^{6} \mathrm{~Hz} \\
(6.3 \mathrm{e}+14)\end{array}$ & 150 & 10 & 215 & $\begin{array}{c}>1,000 \\
\left(R E^{k}=10^{-2}\right)\end{array}$ & $1,621 \mathrm{~s}$. & $>3,658 \mathrm{~s}$. \\
\hline \hline
\end{tabular}

$*+$ the three translational rigid body modes

\section{Conclusions}

The domain decomposition based FETI-DPH iterative solution method introduced in this paper addresses the class of real indefinite problems of the form $(\mathbf{A}-\sigma \mathbf{M}) \mathbf{x}=\mathbf{b}$, where $\mathbf{A}$ and $\mathbf{M}$ are symmetric positive semi-definite and arise from the finite element discretization of second-order elastodynamic problems. It is essentially an extension of the FETI-DP [4,5] method which replaces the PCG solver by the PGMRES solver, and augments the basic coarse problem with the free-space solutions of Navier's homogeneous displacement equations of motion. Preliminary numerical investigations reveal 
that the FETI-DPH method can handle values of $\sigma$ that are large in comparison with the converged eigenvalues of the pencil $(\mathbf{A}, \mathbf{M})$, and that for a given $\sigma$, the FETI-DPH method is numerically scalable with respect to all of the subdomain size, number of subdomains, and problem size. The extension of the FETI-DPH method to fourth-order dynamic plate bending problems is straightforward as the FETI-DP method is readily available as well as numerically scalable for fourth-order plate bending problems, and the free-space solutions of the fourth-order partial differential equation governing the freevibration of thin plates are well-known (for example, see [1]). Current work is focusing on extending the FETI-DPH method to the case where $\mathbf{A}$ and $\mathbf{M}$ arise from the finite element discretization of plate and shell problems.

\section{Acknowledgements}

The first author acknowledges the support by the Sandia National Laboratories under Contract No. 29341. The second author acknowledges the support by the National Science Foundation under Grant No. DMS-0209297. Any opinions, findings, and conclusions or recommendations expressed in this material are those of the authors and do not necessarily reflect the views of the National Science Foundation or the Sandia National Laboratories. Both authors thank Philip Avery, Michel Lesoinne, and Radek Tezaur for their help in the computer implementation of the FETI-DPH method.

\section{A Appendix}

The objective of this appendix is to show that an interplay between a low mesh resolution and the number of wave directions $N_{\theta}$ can cause $\mathbf{Q}_{b}$ to become rank deficient. Hence, this interplay is one among several motivations for filtering the matrix of augmentation coarse modes $\mathbf{Q}_{b}$ as described in Section 3.2.

For simplicity, the following proposition considers the case of a two-dimensional problem, a uniform discretization characterized by a mesh size $h$, and an edge $E$ of the mesh partition that is aligned with the $x$-axis and begins at its origin. In this case,

$$
\theta_{j}=\left[\begin{array}{c}
\cos \vartheta_{j} \\
\sin \vartheta_{j}
\end{array}\right], \quad X_{m}=\left[\begin{array}{c}
x_{m} \\
0
\end{array}\right]=\left[\begin{array}{c}
m h \\
0
\end{array}\right], \quad \theta_{j} \cdot X_{m}=x_{m} \cos \vartheta_{j}
$$

and Eqs. (32) become 


$$
\begin{aligned}
\mathbf{q}_{b_{l+q}}\left[\begin{array}{l}
2(m-1)+1 \\
2(m-1)+2
\end{array}\right] & = \begin{cases}a_{p_{j}} \sin \left(k_{p} x_{m} \cos \vartheta_{j}\right), & q=0, \\
a_{p_{j}} \cos \left(k_{p} x_{m} \cos \vartheta_{j}\right), & q=1, \\
a_{s_{j}} \sin \left(k_{s} x_{m} \cos \vartheta_{j}\right), & q=2, \\
a_{s_{j}} \cos \left(k_{s} x_{m} \cos \vartheta_{j}\right), & q=3,\end{cases} \\
l & =4(j-1)+1, \\
m & =1, \cdots, N_{I}-N_{c} .
\end{aligned}
$$

Proposition Let $k$ denote either a wave number $k_{p}$ associated with a pressure wave, or a wave number $k_{s}$ associated with a shear wave (see Eqs. (30)). If the augmentation coarse problem contains two directions $\theta_{i}$ and $\theta_{j}$ whose angles with the $x$-axis $\vartheta_{i}$ and $\vartheta_{j}$ satisfy

$$
\cos \vartheta_{i} \pm \cos \vartheta_{j}=\frac{n \pi}{k h}
$$

where $n$ is an integer, then

(1) $\vartheta_{i}$ and $\vartheta_{j}$ satisfy

$$
\cos \left(k x_{m} \cos \vartheta_{i}\right)= \pm \cos \left(k x_{m} \cos \vartheta_{j}\right), \quad \forall X_{m} \in E .
$$

(2) The matrix $\mathbf{Q}_{b}$ defined by Eqs. (A.2) is rank deficient.

Proof Since $E$ is aligned with the $x$-axis and begins at its origin, and the mesh is assumed to be uniform, then

$$
\forall X_{m} \in E, x_{m}=m h
$$

From Eq. (A.3) and Eq. (A.5), it follows that

$$
\begin{aligned}
\cos \left(k x_{m} \cos \vartheta_{i}\right) & =\cos \left(k x_{m}\left(\frac{n \pi}{k h} \mp \cos \vartheta_{j}\right)\right) \\
& =\cos \left(\mp k x_{m} \cos \vartheta_{j}+x_{m} \frac{n \pi}{h}\right) \\
& =\cos \left(\mp k x_{m} \cos \vartheta_{j}+(m h) \frac{n \pi}{h}\right) \\
& =\cos \left(\mp k x_{m} \cos \vartheta_{j}+n m \pi\right) .
\end{aligned}
$$

Hence, $\forall X_{m} \in E$,

$$
\cos \left(k x_{m} \cos \vartheta_{i}\right)= \pm \cos \left(k x_{m} \cos \vartheta_{j}\right)
$$

From Eq. (A.2) and the above result, it follows that the matrix $\mathbf{Q}_{b}$ has at least two identical columns and therefore is rank deficient. 
Since $\left|\cos \vartheta_{i} \pm \cos \vartheta_{j}\right| \leq 2$, the result stated in the above proposition is significant when $\frac{2 \pi}{k h} \leq \frac{4}{n}$, and therefore when the mesh resolution is less or equal to 4 elements per wave length $(n=1)$.

\section{References}

[1] M. Géradin and D. Rixen, Mechanical Vibrations: Theory and Application to Structural Dynamics, J. Wiley, 1994.

[2] W. W. Hager, Applied Numerical Linear Algebra, Prentica Hall, New Jersey, 1988.

[3] L. F. Pavarino and A. Toselli, eds., Recent Developments in Domain Decomposition Methods, Lecture Notes in Computational Science and Engineering, Springer, 23:1-26, 2002.

[4] C. Farhat, M. Lesoinne and K. Pierson, A scalable dual-primal domain decomposition method. Numer. Lin. Alg. Appl. (2000) 7:687-714.

[5] C. Farhat, M. Lesoinne, P. LeTallec, K. Pierson and D. Rixen, FETI-DP: a dual-primal unified FETI method - part I: a faster alternative to the two-level FETI method, Internat. J. Numer. Meths. Engrg. (2001) 50:1523-1544.

[6] C. Farhat, A Lagrange multiplier based divide and conquer finite element algorithm, J. Comput. Sys. Engrg. (1991) 2:149-156.

[7] C. Farhat and F. X. Roux, A method of finite element tearing and interconnecting and its parallel solution algorithm, Internat. J. Numer. Meths. Engrg. (1991) 32:1205-1227.

[8] C. Farhat and F. X. Roux, An unconventional domain decomposition method for an efficient parallel solution of large-scale finite element systems, SIAM J. Sc. Stat. Comp. (1992) 13(1):379-396.

[9] C. Farhat, J. Mandel and F. X. Roux, Optimal convergence properties of the FETI domain decomposition method, Comput. Meths. Appl. Mech. Engrg. (1994) 115:367-388.

[10] C. Farhat and J. Mandel, The two-level FETI method for static and dynamic plate problems - part I: an optimal iterative solver for biharmonic systems, Comput. Meths. Appl. Mech. Engrg. (1998) 155:129-152.

[11] C. Farhat, P. S. Chen, J. Mandel and F. X. Roux, The two-level FETI method - part II: extension to shell problems, parallel implementation and performance results, Comput. Meths. Appl. Mech. Engrg. (1998) 155:153-180.

[12] J. Mandel and R. Tezaur, Convergence of a substructuring method with Lagrange multipliers, Numerische Mathematik (1996) 73:473-487. 
[13] J. Mandel, R. Tezaur and C. Farhat, A scalable substructuring method by Lagrange multipliers for plate bending problems, SIAM J. Numer. Anal. (1999) 36:1370-1391.

[14] A. Klawonn and O. Widlund, FETI and Neumann-Neumann iterative substructuring methods: connections and new results, Comm. Pure Appl. Math. (2001) 54:57-90.

[15] M. Bhardwaj, D. Day, C. Farhat, M. Lesoinne, K. Pierson and D. Rixen, Application of the FETI method to ASCI problems: scalability results on onethousand processors and discussion of highly heterogeneous problems, Internat. J. Numer. Meths. Engrg. (2000) 47:513-536.

[16] M. Bhardwaj, K. Pierson, G. Reese, T. Walsh, D. Day, K. Alvin, J. Peery, C. Farhat and M. Lesoinne, Salinas: a scalable software for high-performance structural and solid mechanics simulations, Proc. IEEE/ACM SC2002 Conference, Baltimore, Maryland, November 16-22, 2002.

[17] C. Farhat, P. S. Chen and J. Mandel, A scalable Lagrange multiplier based domain decomposition method for implicit time-dependent problems, Internat. J. Numer. Meths. Engrg. (1995) 38:3831-3858.

[18] C. Farhat, P. S. Chen, F. Risler and F. X. Roux, A unified framework for accelerating the convergence of iterative substructuring methods with Lagrange multipliers, Internat. J. Numer. Meths. Engrg. (1998) 42:257-288.

[19] J. Mandel and R. Tezaur, On the convergence of a dual-primal substructuring method, Numer. Math. (2001) 88:543-558.

[20] A. Klawonn, O. B. Widlund and M. Dryja, Dual-primal FETI methods for three-dimensional elliptic problems with heterogeneous coefficients, SIAM J. Numer. Anal. (2002) 40(1):159-179.

[21] M. Lesoinne, A FETI-DP corner selection algorithm for three-dimensional problems, Proceedings of the 2002 Domain Decomposition Conference, Cocoyoc, Mexico, 2002.

[22] D. Rixen and C. Farhat, A simple and efficient extension of a class of substructure based preconditioners to heterogeneous structural mechanics problems, Internat. J. Numer. Meths. Engrg. (1999) 44:489-516.

[23] Y. Saad, Iterative methods for sparse linear systems, PWS Publishing Company, Boston, 1995.

[24] C. Farhat, A. Macedo and M. Lesoinne, A two-level domain decomposition method for the iterative solution of high frequency exterior Helmholtz problems, Numer.Math. (2000) 85:283-308.

[25] C. Farhat and M. Géradin, On the General Solution by a Direct Method of a Large-Scale Singular System of Linear Equations: Application to the Analysis of Floating Structures, Internat. J. Numer. Meths. Engrg. (1998) 41:675-696.

[26] G. H. Golub and C. F. Van Loan, Matrix Computations, Johns Hopkins, Baltimore, Maryland, 1983. 